\title{
Philosophiques
}

\section{Le sens du projet lévinassien : une spiritualité athée universelle pour un nouveau paradigme?}

\section{Muriel Briançon}

Volume 45, numéro 2, automne 2018

URI : https://id.erudit.org/iderudit/1055268ar

DOI : https://doi.org/10.7202/1055268ar

Aller au sommaire du numéro

Éditeur(s)

Société de philosophie du Québec

ISSN

0316-2923 (imprimé)

1492-1391 (numérique)

Découvrir la revue

Citer cet article

Briançon, M. (2018). Le sens du projet lévinassien : une spiritualité athée universelle pour un nouveau paradigme ? Philosophiques, 45(2), 365-390. https://doi.org/10.7202/1055268ar
Résumé de l'article

Afin d'éclairer le sens de la religiosité de la philosophie de Lévinas et d'écarter le soupçon d'une soumission de celle-ci à des dogmes ou à des croyances incompatibles avec un principe de laïcité, notre contribution vise à clarifier le sens de son projet général. L'intention lévinassienne, ambitieuse et visionnaire, consiste en l'explicitation philosophique, métaéthique et phénoménologique, de l'idée cartésienne de l'Infini, transcendance non idolâtrique surgissant de la relation humaine. Cette quête philosophique de l'extrême conscience suppose un athéisme ou du moins une mise en question de l'idolâtrie, du sacré et des dogmes. Cette spiritualité laïque universelle réalisée dans un judaïsme repensé conduit à des questions philosophiques essentielles et à un changement paradigmatique. 


\title{
Le sens du projet lévinassien : une spiritualité athée universelle pour un nouveau paradigme?
}

\author{
MURIEL BRIANÇON \\ Maître de conférences HDR en sciences de l'éducation \\ UCO La Réunion - Equipe de recherche PESSOA \\ muriel.briancon@ucolareunion.fr
}

\begin{abstract}
RÉSUMÉ. - Afin d'éclairer le sens de la religiosité de la philosophie de Lévinas et d'écarter le soupçon d'une soumission de celle-ci à des dogmes ou à des croyances incompatibles avec un principe de laïcité, notre contribution vise à clarifier le sens de son projet général. L'intention lévinassienne, ambitieuse et visionnaire, consiste en l'explicitation philosophique, métaéthique et phénoménologique, de l'idée cartésienne de l'Infini, transcendance non idolâtrique surgissant de la relation humaine. Cette quête philosophique de l'extrême conscience suppose un athéisme ou du moins une mise en question de l'idolâtrie, du sacré et des dogmes. Cette spiritualité laïque universelle réalisée dans un judaïsme repensé conduit à des questions philosophiques essentielles et à un changement paradigmatique.
\end{abstract}

\begin{abstract}
To enlighten the sense of the religiosity of the Lévinas' philosophy and rule out the suspicion of a submission of this one in dogmae or in incompatible faiths with a principle of secularism, our contribution aims at clarifying the sense of its general project. The Lévinas' intention, ambitious and visionary, consists of the philosophic explicitation, métaéthique and phenomenological, of the Cartesian idea of Infinity, the not idolatrous transcendence appearing from the human relation. This philosophic quest of the extreme consciousness supposes an atheism or at least a questioning of the idolization, the sacred and the dogmae. This universal laic spirituality realized in a rethought Judaism leads to essential philosophic questions and to paradigmatic change.
\end{abstract}

\section{Introduction}

Trop juif pour les non-juifs, pas assez juif pour les juifs, trop religieux pour les laïcs et trop athée pour les croyants, Lévinas est sans place précise (atopos) ou au contraire Au carrefour de la foi et de la raison ${ }^{1}$, ce lieu peu commode et très controversé ${ }^{2}$. D'aucuns rappellent qu'il y a corrélation entre philosophie et judaïsme dans la pensée de Lévinas³. Or, tant que sa

1. Notre traduction du titre de l'ouvrage de Denise Egéa-Kuehne, Lévinas and Education. At the intersection of Faith and Reason, New York, Ed. Egéa-Kuehne, 2008.

2. Voir également la critique d'une "embardée" voire d'une "prise en otage théologique " de la phénoménologie française par Dominique Janicaud, dans La phénoménologie dans tous ses états, Paris, Gallimard, I990, rééd. 2009, p. 64 et 74.

3. David Banon, "Levinas, penseur juif ou juif qui pense ", Noesis [en ligne], $\mathrm{n}^{\circ} 3,2000$ : URL: http://noesis.revues.org/7.

Fabio Ciaramelli, «Le rôle du judaïsme dans l'œuvre de Levinas ", Revue philosophique de Louvain, $\mathrm{n}^{\circ}$ 52, 1983, p. 580-600. 
pensée sera corrélée au judaïsme, donc soupçonnée d'incompatibilité avec les valeurs de la République, celle de la laïcité notamment, l'extra-ordinaire (dans tous les sens du terme) éthique humaniste proposée par Lévinas sera contestée. Ce soupçon d'une incompatibilité entre religion et laïcité qui pèse particulièrement sur l'islam ${ }^{4}$ se fait jour également à propos du judaïsme ${ }^{5}$.

Certes, une première lecture trop rapide de l'œuvre imposante et difficile d'accès, à la fois philosophique et talmudique, de Lévinas, peut donner l'impression d'une confusion entre le philosophique et le religieux. Mais nous faisons au contraire l'hypothèse que cette ambigüité — loin d'être accidentelle - provient de l'intention même de Lévinas qui était justement de redéfinir ces catégories, pour remanier leurs liens et ainsi proposer un nouveau paradigme éthique universel et laïque, dont nous avons aujourd'hui cruellement besoin en philosophie de l'éducation. Cette contribution se propose donc d'expliciter encore et encore l'ambitieux projet lévinassien, afin d'en permettre une meilleure compréhension et utilisation dans le champ éducatif.

\section{Le cœur du projet lévinassien: une explicitation de l'idée cartésienne de l'infini}

Pour plus de clarté didactique, livrons une vue d'ensemble du projet de Lévinas, que nous présenterons en seize étapes successives, en espérant que le lecteur nous pardonnera cette longue mais nécessaire démonstration: Lévinas veut expliciter philosophiquement - par une philosophie de la rupture et de l'amour, plus précisément métaphysique voire métaéthique et grâce à une phénoménologie non intentionnelle — l'idée cartésienne de l'Infini, qui est une transcendance non religieuse et le sens même du mot «Dieu », idée surgissant de la relation éthique et menant à la gloire et au divin jusqu'à la sainteté, mais contraire aux dogmes religieux, au sacré et aux mythes, redéfinissant ainsi la catégorie de religion — proche d'un athéisme et d'une spiritualité universelle réalisée dans le judaïsme, lui-même redéfini comme extrême conscience - et conduisant à des questions philosophiques essentielles grâce à une méthode: l'herméneutique talmudique.

\section{Une explicitation philosophique}

L'intention de Lévinas est en effet de réaliser une explicitation philosophique. Il s'inscrit dans la lignée des philosophes occidentaux en s'y référant continuellement: "Lévinas appartient à la haute tradition philosophique occidentale qui part de Platon pour arriver à Bergson, Husserl et Hei-

4. Abdennour Bidar, "Islam et valeurs de la République ", Administration et Education, $\mathrm{n}^{\circ} 4$, 20I5, p. 95-IO4.

5. Laurent Klein, "Judaïsme, valeurs républicaines et laïcité ", Administration et Education, $\mathrm{n}^{\circ} 4$, 20I 5, p. I05-I09. 
degger ${ }^{6}$.» Même si son positionnement philosophique est critique, l'auteur se réfère très souvent à ses prédécesseurs et à leurs concepts: le Bien au-delà de l'essence de Platon, l'Un au-delà de l'être de Plotin, l'idée de l'infini de Descartes, la phénoménologie husserlienne et l'il y a heideggérien, pour ne citer qu'eux. D'ailleurs, l'apport de cette démarche est reconnu par les juifs eux-mêmes: «L'explicitation philosophique du judaïsme par Lévinas ramène au judaïsme Juifs et non-Juifs qui s'inclineraient devant les textes pour établir un dialogue avec eux et en attendre des enseignements et des orientations ${ }^{7}$." Ainsi la pensée lévinassienne restitue-t-elle sa dimension proprement philosophique à la tradition juive.

\section{Par une philosophie de la rupture et de l'amour}

Mais de quelle philosophie s'agit-il ? La philosophie de Lévinas est d'abord une philosophie de la rupture, car sa pensée éthique remet totalement en question la philosophie traditionnelle et renverse jusqu'à la manière même de philosopher: "la force de rupture de l'éthique n'atteste pas un simple relâchement de la raison, mais une mise en question du philosopher, laquelle ne peut pas retomber en philosophie. Mais quel singulier retournement ! ${ }^{8}$ Lévinas distingue en effet la philosophie (histoire des idées philosophiques) du philosopher (réfléchir philosophiquement). Cette rupture serait une "fêlure », une "brisure " ${ }^{9}$, dont Lévinas est conscient et dont il accepte l'inhérente difficulté: "tant pis pour la marche sous un soleil sans ombre que serait la philosophie ${ }^{10}$.

Cette rupture philosophique se réalise sur plusieurs plans:

- Rupture d'avec cette philosophie [occidentale d'origine grecque] insatisfaite d'elle-même, caractérisée par son désir de savoir et son obsession d'apporter des réponses: "Philosophie, toujours insatisfaite de n'être que philosophie $!^{11}$ » est celle d'une conscience malheureuse qui ne cherche que des réponses: "philosophie de la réponse - c'est la réponse qui compte; c'est le résultat comme dit Hegel ${ }^{12}$.

- Rupture d'avec sa dimension ontologique car le savoir désiré est corrélé à l'être: "en tant que savoir, la pensée porte sur le pensable; sur le pensable appelé être. Portant sur l'être, elle est hors d'elle-même, mais demeure merveilleuse-

6. Mickaël de Saint Chéron, Entretiens avec Emmanuel Lévinas 1983-1994, Paris, LGB, coll. «Livre de poche», 2006, rééd. 2010, p. 62.

7. Ami Bouganim, "Lévinas pédagogue", in Emmanuel Lévinas, philosophe et pédagogue, Paris, Ed. du Nadir de l'Alliance israélite universelle, I998, p. 63.

8. Emmanuel Lévinas, De dieu qui vient à l’idée, Paris, Vrin, I982, p. I9.

9. Salomon Malka, Lévinas, la vie et la trace, Paris, Albin Michel, 2002, rééd. 2005, p. 267.

10. Lévinas, De dieu qui vient à l'idée, p. I43.

11. Emmanuel Lévinas, Altérité et transcendance, Saint Clément de rivière, Fata Morgana, I995, p. 32 .

12. Ibid., p. I36. 
ment en elle-même ou revient à elle-même» dit Lévinas dans ses entretiens ${ }^{13}$. Cette remise en question du primat de l'ontologie est " une téméraire entreprise » que Lévinas entreprend dès I95 I dans son article "L'ontologie est-elle fondamentale? $»^{14}$.

- Rupture d'avec l'immanence, ce qui est explicitement écrit dans le premier chapitre de De Dieu qui vient à l'idée ${ }^{15}$ : Lévinas s'oppose à cette "philosophie qui juge la transcendance hors de portée de l'être pensant mais fini », qui ne considère l'infini que comme " une idée méthodologique », " un principe régulateur» du progrès scientifique, une «Idée et rien qu'idée, sans contrepartie dans l'être ${ }^{16}$.

- Rupture d'avec ses prédécesseurs, car «Lévinas rompt avec une tradition philosophique qui, de Platon à Hegel, ramenait l'Autre au Même dans le savoirpouvoir de la pensée ${ }^{17}$ ». Lévinas se situe "aux antipodes du spinozisme ${ }^{18}$ », critique Hegel et s'oppose à l'ontologie heideggérienne.

S'il rejette aussi fortement la philosophie occidentale, c'est pour prôner un autre type de philosophie, en retrouvant le sens étymologique de

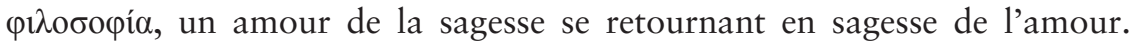
Lévinas se réfère en effet à Plotin pour définir la vraie philosophie opposée à une philosophie du savoir : "Cette nostalgie, ou cette piété ou ce recueillement allant au-delà et au-dessus de l'intelligible présent à l'intelligence, est philosophie, aspiration à une sagesse, qui n'est pas savoir, qui n'est pas représentation, qui est amour. Amour d'une sagesse autre que l'intelligible se donnant au savoir ${ }^{19}$.»

L'amour n'est pas tant désir, manque, aspiration qu' "amour sans concupiscence, cher à Pascal, un amour de l'âme, qui appelle une communion transcendante ${ }^{20}$ ». Philosophie de l'amour, la pensée lévinassienne est tout entière tournée vers l'idéal platonicien du Bien: "La Place du Bien, audessus de toute essence, est l'enseignement le plus profond — l'enseignement définitif — non pas de la théologie, mais de la philosophie ${ }^{21}$. »

13. François Poirié, Emmanuel Lévinas. Essai et entretiens, Arles, Actes Sud, I987, rééd. I996, p. I76.

14. Emmanuel Lévinas, Entre nous. Essais sur le penser-à-l'autre, Paris, Grasset \& Fasquelle, I99I, p. I2.

15. Lévinas, De dieu qui vient à l'idée.

16. Lévinas, Altérité et transcendance, p. $7 \mathrm{I}$.

17. Poirié, Emmanuel Lévinas. Essai et entretiens, p. 57.

18. Emmanuel Lévinas, Totalité et infini. Essai sur l'extériorité, La Haye, Martinus Nijhoff, I96I, rééd. I97I, p. I07.

19. Lévinas, Altérité et transcendance, p. 3 I.

20. De Saint Chéron, Entretiens avec Emmanuel Lévinas 1983-1994, p. 20.

21. Lévinas, Totalité et infini, p. Io6. 


\section{Plus précisément métaphysique voire métaéthique}

Tout en définissant le but de ses recherches, il insiste sur le fait qu'elles ne sont pas théologiques ou religieuses mais bien philosophiques, et plus précisément métaphysiques, car au-dessus ou au-delà de la physique (la nature, l'être, l'ontologie): "Il serait faux de la [la relation métaphysique à l'idée de l'infini] qualifier de théologique. [...] Décrire cette relation constitue le thème même de ces recherches ${ }^{22}$. " Lévinas définit donc son projet métaphysique comme une troisième voie entre religion et philosophie occidentale:

Entre une philosophie de la transcendance qui situe ailleurs la vraie vie [...] et une philosophie de l'immanence où l'on se saisirait véritablement de l'être quand tout «autre» (cause de guerre), englobé par le Même, s'évanouirait au terme de l'histoire, nous nous proposons de décrire, dans le déroulement de l'existence terrestre, de l'existence économique comme nous l'appelons, une relation avec l'Autre, qui n'aboutit pas à une totalité divine ou humaine, une relation qui n'est pas une totalisation de l'histoire, mais l'idée de l'infini. Une telle relation est la métaphysique même ${ }^{23}$.

Métaphysique radicale ${ }^{24}$ ou métaéthique ${ }^{25}$, la philosophie de la rencontre humaine de Lévinas relève de la phénoménologie.

\section{Grâce à une phénoménologie non intentionnelle}

Lévinas se revendique en effet de la phénoménologie:

Je pense que, malgré tout, ce que je fais, c'est de la phénoménologie, même s'il n'y a pas de réduction selon les règles exigées par Husserl, même si toute la méthodologie husserlienne n'est pas respectée. [...] La phénoménologie ce n'est pas ériger les phénomènes en choses en soi; c'est ramener les choses en soi à l'horizon de leur apparaître, de leur phénoménalité, à faire apparaître l'apparaître lui-même derrière la quiddité qui apparaît, même si cet apparaître n'incruste pas ses modalités dans le sens qu'il livre au regard. [...] Tous ceux qui pensent ainsi et cherchent ces dimensions pour trouver ce sens font de la phénoménologie ${ }^{26}$.

Cet ancrage phénoménologique serait d'ailleurs, peut-être, l'une des raisons de la tardive consécration universitaire de Lévinas: "Pour J.-L. Marion [...] sa philosophie était considérée comme très difficile, parce que c'était de la phénoménologie à l'état pur par quelqu'un qui la connaissait depuis le début ${ }^{27}$.» Lévinas reconnaît d'ailleurs la dette qu'il a à l'égard du père de la phénoménologie qu'il a traduit et contribué à faire connaître en

22. Ibid., p. 32.

23. Ibid., p. 44 .

24. Joseph Habib Cohen, Stéphane \& Raphaël Zagury-Orly, «Emmanuel Lévinas: la métaphysique radicale ", Les Temps modernes, $\mathrm{n}^{\circ} 664$, $20 \mathrm{II}$, p. I70-I93.

25. De Saint Chéron, Entretiens avec Emmanuel Lévinas 1983-1994, p. 73.

26. Lévinas, De dieu qui vient à l'idée, p. I40.

27. Malka, Lévinas, la vie et la trace, p. 279. 
France: "C'est sans doute Husserl qui est à l'origine de mes écrits ${ }^{28}$ ", dit-il. Sans jamais avoir recours à la Lebensphilosophie ni au vitalisme, si répandus dans les années 30 et 40 en Allemagne, il considère que la réduction transcendantale husserlienne conduit à un éveil de la conscience et de la vie: «C'est la vie sous-jacente au regard que la phénoménologie husserlienne réveille. [...] Il s'agit, dans la présence, de retrouver la vie ${ }^{29}$."

Cependant, tout en restant dans le cadre phénoménologique et après avoir été marqué par la Phénoménologie de l'esprit de Hegel et Être et temps d'Heidegger, Lévinas s'oppose farouchement à ce primat de l'ontologie qui domine la pensée occidentale. Tout en reprenant le concept d'intentionnalité (représentation, thématisation, acte objectivant) de son maître, Lévinas préfère revenir à une conscience pré-réflexive non intentionnelle: "L'interpellation [éthique] ne me fait-elle pas entrer dans une pensée non intentionnelle de l'in-saisissable $?^{30} »$.

\section{De l'idée cartésienne de l'infini}

Cet insaisissable, «idée exceptionnelle, idée unique [...] Pensée qui, dans sa phénoménologie, ne se laisse pas réduire, sans reste, à l'acte de conscience d'un sujet, à la pure intentionnalité thématisante ${ }^{31}$ » est l'idée-de-l'infini-ennous, que Lévinas emprunte à Descartes. Dans sa Troisième Méditation en effet, ce dernier avait tenté de démontrer que le cogito cartésien possédait l'idée de Dieu, «substance infinie, éternelle, immuable, indépendante, toute connaissante, toute puissante ${ }^{32} »$. Mais, pour Lévinas, une pensée qui pense plus qu'elle ne pense n'est pas possession mais Désir. C'est donc à réaliser une phénoménologie de l'idée de l'Infini - qui n'intéressait pas Descartes - que va s'attacher Lévinas et en cela que va résider son projet:

L'idée de l'Infini enseignée dans son paradoxe par Descartes, pensée sans pareille, pensant plus qu'elle ne peut contenir dont nous avons essayé de dire la sagesse concrète dans l'obéissance au commandement qui, dans le visage d'autrui, me voue à l'autre homme — voilà de la «visée du futur », par-delà l'à-venir, la vraie "phénoménologie». Pensée pensant plus qu'elle ne pense ou pensée qui, en pensant, fait mieux que de penser $[\ldots]^{33}$.

Lévinas précise d'abord ce que l'idée de l'infini n'est pas:

- L'infini n'est pas une pensée immanente du cogito cartésien: "Si penser consiste à se référer à un objet, il faut croire que la pensée de l'infini n'est pas une pensée $\mathrm{e}^{34}$.»

28. Poirié, Emmanuel Lévinas. Essai et entretiens, p. I73.

29. Lévinas, De dieu qui vient à l'idée, p. 53.

30. Poirié, Emmanuel Lévinas. Essai et entretiens, p. I87.

31. Lévinas, De dieu qui vient à l'idée, p. 9.

32. Descartes, Les méditations métaphysiques, Paris, Bordas, rééd. I987, [22].

33. Lévinas, Altérité et transcendance, p. 55.

34. Lévinas, Totalité et infini, p. 232. 
— «L'infini n'est pas objet d'une connaissance», mais «ce qui est approchable par une pensée qui à tout instant pense plus qu'elle ne pense ${ }^{35}$ ».

Que recouvre alors cette idée de l'infini ? Pour Lévinas qui s'oppose à Descartes, l'idée de l'infini implique séparation et extériorité: "L’idée de l'Infini demande cette séparation ${ }^{36}$ " et "son extériorité totale par rapport à celui qui le pense ${ }^{37}$ ». Il s'agit d' " une expérience morale concrète ${ }^{38}$ », de "l'infinition de l'infini ${ }^{39}$ ", $d$ ' " une hauteur, une noblesse, une transcendance ${ }^{40}$ ».

\section{Qui est une transcendance non religieuse et le sens même du mot «Dieu»}

L'idée de l'infini signifie donc transcendance: «Le concept de transcendance, rigoureusement développé, s'exprime par le terme de l'infini ${ }^{41}$. » Or la transcendance serait une, voire la principale problématique de la philosophie:

Transcendance de l'idée cartésienne de l'Infini dans une pensée qui se trouve penser plus qu'elle ne saurait embrasser, éblouissement du regard par un surplus de lumière et éclatement du savoir en adoration auxquels fait allusion Descartes à la fin de la troisième Méditation. [...] Idée exceptionnelle de l'Infini évadée de l'être, de la présence plus forte et plus vénérable que la totalité. Idée qu'on ne saurait en vertu de "l'argument ontologique» reléguer sans autre forme de procès dans la présence, dans l'être enfermé dans le regard totalisant, ni dans un quelconque arrière-monde, ni dans quelque ciel-vide. La recherche du lieu originel de cette idée de l'Infini et de sa transcendance est sans doute l'un des problèmes principaux de la philosophie ${ }^{42}$.

En élaborant cette phénoménologie non intentionnelle de l’idée de l'infini, en liant le concept cartésien à la notion de transcendance, Lévinas reste-t-il dans la philosophie ou «a-t-il imprimé à la phénoménologie un religious turn comme il y a eu un linguistic turn en philosophie ${ }^{43}$ ", " un tournant théologique dans le domaine de la phénoménologie ${ }^{44}$ » voire " une prise en otage théologique ${ }^{45}$ »?

La position de Lévinas est très précise sur ce point. La transcendance lévinassienne n'a rien de religieux puisqu'elle rejette l'intentionnalité, le savoir, la représentation, la thématisation, la participation, la totalité, qui

35. Ibid., p. 232.

36. Ibid., p. I04.

37. Ibid., p. 42.

38. Ibid., p. 46.

39. Ibid., p. I 2.

40. Ibid., p. 3 I.

41. Ibid., p. Iо.

42. Lévinas, Altérité et transcendance, p. 28.

43. Banon, "Levinas, penseur juif ou juif qui pense », p. 7.

44. Jeffrey Andrew Barash, "Emmanuel Lévinas: penseur du religieux?", Bulletin du Centre de recherche français à Jérusalem, $\mathrm{n}^{\circ} \mathrm{I} 8,2007$, p. II 7 .

45. Dominique Janicaud, La phénoménologie dans tous ses états, Paris, Gallimard, I990, rééd. 2009, p. 74. 
sont des principes imposés par la religion, et qu'elle questionne Dieu philosophiquement:

Rechercher «l'origine» du mot Dieu, les circonstances concrètes de sa signifiance, est absolument nécessaire. On commence par accepter sa Parole au nom de l'autorité sociale de la religion. Comment être sûr que la Parole ainsi acceptée est bien celle que parle Dieu? Il faut rechercher l'expérience originelle. La philosophie — ou la phénoménologie — est nécessaire pour reconnaître Sa voix ${ }^{46}$.

Nous comprenons que Lévinas a tenté de construire une phénoménologie de la transcendance, dans laquelle se retrouvent aujourd'hui certains philosophes ${ }^{47}$. Ce que recherche Lévinas en déployant cette phénoménologie, c'est le sens humain de l'idée de l'infini, transcendance ou parole de Dieu. Sa problématique est explicite: "L'infini actuel a-t-il un sens? Équivaut-il à l'être même? Ou n'est-il qu'une idée régulatrice? N'est-il qu'un simple mot ? $^{48}$ » Quel est pour l'être humain le sens du mot Dieu?

\section{Surgissant de la relation éthique}

C’est dans une phénoménologie de la relation et du dialogue faisant référence à Gabriel Marcel et Martin Buber que Lévinas trouve le sens de cette transcendance non religieuse, ce Dieu non thématisé, non représenté:

Extra-ordinaire relation [Je-Tu]. Dès la première description que Buber et Marcel en font, le mot Dieu est prononcé comme s'il éclairait l'espace où la rectitude du dialogue peut se dessiner. Toi par excellence. Toi «éternel », s'offrant à l'invocation plutôt qu'à la constatation et à l'expérience, Dieu invisible ${ }^{49}$.

En effet, la transcendance surgit de la relation sociale et du face-à-face intersubjectif: "La transcendance, c'est ce qui nous fait face ${ }^{50}$ ", écrit-il dès I954, et plus tard: "Il faut faire œuvre de justice - la droiture du face-àface - pour que se produise la trouée qui mène à Dieu ${ }^{51}$. . Ce chemin direct qui conduit à Dieu, c'est "l'un-pour-l'autre éthique ${ }^{52}$ », ou plus exactement sa possibilité: "c'est cette rupture de l'indifférence — de l'indifférence fûtelle statistiquement dominante - , la possibilité de l'un-pour-l'autre, qui est l'événement éthique ${ }^{53}$. Cette responsabilité pour l'autre est une préoccupation de l'autre qui va jusqu'à la possibilité idéale d'un sacrifice de soi.

46. Lévinas, Altérité et transcendance, p. I76.

47. Sophie Nordmann, Phénoménologie de la transcendance, Dol de Bretagne, Éd. d'Écarts, 20I 2.

48. Lévinas, Altérité et transcendance, p. 74.

49. Ibid., p. I05.

50. Lévinas, Entre nous. Essais sur le penser-à-l'autre, p. 45.

51. Lévinas, Totalité et infini, p. 77.

52. Lévinas, De dieu qui vient à l'idée, p. I 25.

53. Lévinas, Entre nous. Essais sur le penser-à-l'autre, p. ıо. 
L'originalité et la puissance de la pensée lévinassienne sont d'affirmer que «ce primat de l'éthique, c'est-à-dire de la relation d'homme à homme - signification, enseignement et justice —, primat d'une structure irréduc-

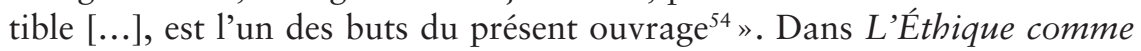
philosophie première, il prône «une sagesse plus urgente ${ }^{55}$ » que l'ontologie. Antérieure, «l'éthique n'est pas du tout une couche qui vient recouvrir l'ontologie, mais ce qui est en quelque façon, plus ontologique que l'ontologie, une emphase de l'ontologie ${ }^{56} »$.

La relation éthique n'a rien d'une nouvelle mystique religieuse:

La relation éthique, le face-à-face tranche aussi sur toute relation qu'on pourrait appeler mystique et où d'autres événements que celui de la présentation de l'être original viennent bouleverser ou sublimer la sincérité pure de cette présentation, où d'enivrantes équivoques viennent enrichir l'univocité originelle de l'expression, où le discours devient incantation comme la prière qui devient rite et liturgie, où les interlocuteurs se trouvent jouer un rôle dans un drame qui a commencé en dehors d'eux ${ }^{57}$.

L'éthique serait plutôt une perspective qui se développe dans la métaphore du voir: «L'éthique n'est pas le corollaire de la vision de Dieu, elle est cette vision même. L'éthique est une optique ${ }^{58}$. " Cette vision se fait à travers le Visage d'autrui qui rappelle l'idée de l'infini: "Vigilance d'une responsabilité qui, de moi à l'autre, est transcendance [...]. Le visage du prochain n'est-il pas le lieu originel où la transcendance appelle une autorité par une silencieuse voix où Dieu vient à l'idée ? Lieu originel de l'Infini ${ }^{59}$. " La transcendance s'exprime alors dans les termes de la relation éthique (proximité, responsabilité, substitution, otage, dés-inter-essement, humanité):

Que la transcendance soit proximité, que la proximité soit responsabilité pour l'autre, substitution à l'autre, expiation pour l'autre, condition — ou incondition — d'otage; $[\ldots]$ voilà quelques termes éthiques par lesquels signifie la transcendance en guise d'humanité ou l'extase comme des-intéressement ${ }^{60}$.

Jusqu'où nous conduit cette optique éthique?

\section{Menant à la gloire et au divin}

La transcendance surgissant de la relation éthique mènerait à la gloire et au divin, non pas au sens chrétien mais au sens pratique. Que signifient en effet

54. Lévinas, Totalité et infini, p. 77.

55. Emmanuel Lévinas, Éthique comme philosophie première, Paris, Payot, I992, rééd. I998, p. 77.

56. Lévinas, De dieu qui vient à l'idée, $\mathrm{p}$. I43.

57. Lévinas, Totalité et infini, p. 222.

58. Emmanuel Lévinas, Difficile liberté. Essais sur le judaïsme, Paris, LGF, I963, rééd. I984, p. 37.

59. Lévinas, Altérité et transcendance, p. 29.

60. Lévinas, De dieu qui vient à l'idée, p. 33. 
ces deux termes? Dans le vocabulaire lévinassien, la gloire est la parole de Dieu, au sens strict d'interpellation éthique et d'assignation à responsabilité:

Retournement à partir du visage d'autrui où, au sein même du phénomène, dans sa lumière même, signifie un surplus de signifiance qu'on pourrait désigner comme gloire. Elle me demande, me réclame, m'assigne. Ne devrait-on pas appeler parole de Dieu, cette demande ou cette interpellation ou cette assignation à responsabilité ?61.

L'appel de «Dieu», c'est l'appel du réfugié, l'appel du sans-abri, l'appel de toute personne en situation de précarité. Pour Lévinas, Dieu est le sens originel qui se dérobe à la représentation et au savoir mais qui se manifeste et signifie dans le Visage:

L'idée importante quand j'évoque le visage d'autrui, la trace de l'Infini, ou la Parole de Dieu, est celle d'une signifiance de sens qui originellement, n'est pas thème, n'est pas objet d'un savoir, n'est pas être d'un étant, n'est pas représentation. Un Dieu qui me concerne par une Parole exprimée en guise de visage de l'autre homme, est une transcendance qui ne devient jamais immanence. Le visage d'autrui est sa manière de signifier. J'emploie aussi une autre formule: Dieu ne prend jamais corps ${ }^{62}$.

L'une des différences, d'une importance extrême, d'avec le christianisme est alors que "Dieu» n'a d'autre corps que celui d'autrui. "Il» ne s'incarne, ni ne vient, jamais - et c'est cela, en quelque sorte, sa "définition» même. Ainsi la transcendance lévinassienne ne signifie-t-elle absolument pas «Dieu» au sens courant et religieux du terme. Lévinas répète d'ailleurs souvent: "le ciel est vide» et "Dieu n'est pas dans le ciel ${ }^{63}$ ". Il récuse également l'idée que l'humain ait été créé à l'image de Dieu, ou inversement que Dieu soit personnifié:

Il serait opportun cependant de demander ici s'il s'agit d'une transcendance à

Dieu ou d'une transcendance à partir de laquelle un mot tel que Dieu révèle seulement son sens. Que cette transcendance se soit produite à partir de la relation (horizontale?) avec autrui, ne signifie ni que l'autre homme soit Dieu, ni que Dieu soit un grand Autrui ${ }^{64}$.

Le projet lévinassien est en effet explicitement de «le dire tel qu' ${ }^{65}$ apparaît, de derrière les concepts dont les plus pieux l'ont déjà déformé et trahi ${ }^{66}{ }$, donc de «renouveler philosophiquement l'advenue de Dieu à l'es-

61. Lévinas, Altérité et transcendance, p. 47.

62. Ibid., p. I7I.

63. De Saint Chéron, Entretiens avec Emmanuel Lévinas 1983-1994, p. I9 et p. 28.

64. Lévinas, De dieu qui vient à l'idée, p. I70.

65. Il faut garder à l'esprit que ce «Il» est un concept qui désigne n'importe quelle personne en situation de besoin ou qui s'approche de «moi».

66. Lévinas, Difficile liberté, p. 283. 
prit $[\ldots]^{67}$ ", ce qui se matérialise en I 982 avec la publication de De Dieu qui vient à l'idée dont Lévinas dit qu'il est «l'un de mes livres sur la philosophie religieuse ${ }^{68} »$.

Voyons tout d'abord ce que ce «Dieu» lévinassien n'est pas:

- Dieu n'est pas une "personnification allégorique de ma conscience morale. $[\ldots]$ nous voilà loin de la voie royale de la piété traditionnelle ${ }^{69}$ ».

- Dieu n'est pas non plus une pensée, puisque justement "l'idée de Dieu fait éclater la pensée laquelle [...] ne fait qu'enfermer en une présence, re-présente, ramène à la présence ou laisse être ${ }^{70} »$. «Dieu » est donc la pensée paradoxale de l'impensable: l'anti-pensée.

— Dieu est encore moins un élément de l'ontologie: «La transcendance de Dieu ne peut ni se dire ni se penser, en termes de l'être, élément de la philosophie [classique], derrière lequel la philosophie ne voit que nuit ${ }^{71}$. Lévinas cherche à voir le noir de la nuit.

- Dieu n'est pas non plus une totalité fermée mais un infini ouvert absolument séparé de l'être humain qui le pense: «Un infini qui ne se ferme pas circulairement sur lui-même [totalité], mais qui se retire de l'étendue ontologique pour laisser une place à un être séparé, existe divinement ${ }^{72}$.»

Listons maintenant six définitions complémentaires données par Lévinas pour Dire «Dieu », la première étant sans conteste la plus importante:

- Dieu est la courbure de l'intersubjectivité: « Cette courbure de l'espace [intersubjectif] est peut-être la présence même de $\operatorname{Dieu}^{73}$ » et "par ma relation avec autrui, je suis en rapport avec Dieu ${ }^{74} »$.

- Dieu est l'infini, conformément au sens de la kabbale médiévale (En Sof = in-fini) et au sens cartésien: "actuellement infini en un si haut degré qu'il ne se peut rien ajouter à la souveraine perfection qu'il possède ${ }^{75}$ »

- Dieu est le tiers convoqué par le Visage et devant lequel le sujet est responsable: "C'est aussitôt le rapport de responsabilité et par conséquent la parole de Dieu. [...] On est trois quand on est deux. [...] Cette place de troisième est unique. Dieu est présent. C'est déjà à lui qu'on répond ${ }^{76}$.»

67. De Saint Chéron, Entretiens avec Emmanuel Lévinas 1983-1994, p. 73.

68. Ibid., p. 50.

69. Lévinas, Entre nous. Essais sur le penser-à-l'autre, p. 32.

70. Lévinas, De dieu qui vient à l'idée, p. 105.

71. Ibid., p. I25.

72. Lévinas, Totalité et infini, p. I07.

73. Ibid., p. 324 .

74. Lévinas, Difficile liberté, p. 37.

75. Lévinas, Altérité et transcendance, p. 70.

76. De Saint Chéron, Entretiens avec Emmanuel Lévinas 1983-1994, p. 5 I. 
- Dieu est " une suprême hétéronomie et [que] la force qui produit de si merveilleux effets, la force qui institue la force, la force civilisatrice, s'appelle Dieu $^{77}$ ».

— Dieu est une absence de corrélation: «Relation sans corrélation ou amour du prochain qui est amour sans eros. Pour-l'autre homme et par là à-Dieu! [...] origine concrète ou situation originaire où l'Infini se met en moi, où l'idée de l'Infini commande l'esprit et le mot Dieu vient sur le bout de la langue ${ }^{78}$."

— Enfin, Dieu est l'absence de contenu: «l'idée de Dieu, comme signifiant le non-contenu par excellence ${ }^{79} »$.

S'interroger sur le sens du mot Dieu, c'est être en quête de sens: "Je pense que Dieu n'a pas de sens en dehors de la recherche de Dieu ${ }^{80}$. " Dieu est la quête même. Dans cette quête, la relation éthique au non-contenu se manifestant (le divin) est nommée «sainteté».

\section{Jusqu'à la sainteté}

Lévinas exprime en termes de sainteté ${ }^{81}$ cette relation éthique au divin, opérant à partir des vingt dernières années une "incommensurable fracture ${ }^{82}$ » d'après M. de Saint Cheron : "la primauté du saint sur le sacré » et "l'effraction du saint et de la sainteté dans son discours, c'est cela la grande nouveauté qui vient en quelque manière parachever sa pensée, son œuvre ${ }^{83}$ ». D’après lui, la philosophie lévinassienne a courageusement sorti la sainteté du domaine de la transcendance religieuse pour l'introduire dans le domaine de la raison pratique et postkantienne, du concept et de la métaphysique, même si ce transfert suscite des réactions de rejet:

Cette volonté d'introduire la sainteté au rang des catégories philosophiques est l'aboutissement, le parachèvement de cette conscience qui habita si longtemps Lévinas. Question si souvent édulcorée, voire critiquée par quelques philosophes méprisants des champs qu'ils ignorent ou qu'ils relèguent en marge de la philosophie - sans même parler de ceux qui, sous couvert de leurs critiques, font encore allégeance à l'antisémitisme, jusqu'à taxer de nonphilosophe un philosophe d'origine ou d'appartenance juive ${ }^{84}$.

Comme pour Dieu, la sainteté a selon Lévinas de multiples sens complémentaires, dont le premier est central:

77. Lévinas, Difficile liberté, p. 28.

78. Lévinas, De dieu qui vient à l'idée, $\mathrm{p}$. I3.

79. Ibid., p. I05.

80. Ibid., p. I 50.

81. Ne pas comprendre la "sainteté» comme autre chose que la possibilité de donner, spontanément, voire intensément, à autrui.

82. De Saint Chéron, Entretiens avec Emmanuel Lévinas 1983-1994, p. 6I.

83. Ibid., p. 6I-62.

84. Ibid., p. 75 . 
— Elle est responsabilité: «La sainteté est cette responsabilité que j'évoque ${ }^{85}$ », dit Lévinas.

— Elle est exagération: "Cet excès, Lévinas le nommait amour, cette hyperbole, il la nommait sainteté ${ }^{86}$.»

— Elle est l'ouverture devant la justice et la mort: "L'ultime sainteté consiste à accepter la justice ou la mort sans résistance, accepter ce néant et cependant tout de même avoir ce reflet de bonté, de valeur ${ }^{87}$. »

— Elle signifie la condition d'otage: "Le mot "sainteté" est plus flatteur que le mot "otage" ${ }^{88}$."

— Elle est «la seule valeur incontestable ${ }^{89}$ " «dans la certitude qu'il faut laisser à l'autre en tout la première place ${ }^{90} »$.

— Elle est une utopie: "L'idéal de sainteté commande utopiquement notre être ${ }^{91}$. »

—Elle caractérise l'humanité: "L'humain, c'est cette possibilité de sainteté ${ }^{92}$.»

Ainsi la sainteté doit-elle être pensée comme un idéal humaniste, « hors de toute idée de foi religieuse, de transcendance "révélée", en ce qu'elle est sa propre transcendance », " une transcendance non religieuse ${ }^{93}$ ».

\section{Contraire aux dogmes religieux, au sacré et aux mythes}

La pensée lévinassienne ne cesse en effet de dénoncer les dogmes, le sacré, les mythes et l'idolâtrie de toutes les religions. Elle n'accepte ni ne véhicule ellemême aucun contenu dogmatique: "Cette révélation de l'infini ne mène à l'acceptation d'aucun contenu dogmatique ${ }^{94}$." Un étudiant le confirme: "Il m'apporta, en même temps qu'une approche radicalement nouvelle de la philosophie, philosophie qui s'intéresse à autre chose qu'à l'Être au-dessus de tout, qu'à l'ontologie fondamentale, un enseignement résolument "non dogmatique" au moment où j’effectuais mes premiers pas dans le judaïsme recouvré $[\ldots]^{95}$.»

85. Ibid., p. 29.

86. Ibid., p. I6.

87. Ibid., p. 3 I.

88. Ibid., p. 38 .

89. Lévinas, Altérité et transcendance, p. I I9.

90. Poirié, Emmanuel Lévinas. Essai et entretiens, p. I05.

91. Ibid., p. I 16.

92. Ibid., p. II9.

93. De Saint Chéron, Entretiens avec Emmanuel Lévinas 1983-1994, p. I3.

94. Lévinas, Totalité et infini, p. Io.

95. De Saint Chéron, Entretiens avec Emmanuel Lévinas 1983-1994, p. I I. 
L'éthique lévinassienne s'oppose également au sacré: «La relation éthique se définit, contre toute relation avec le sacré, en excluant toute signification qu'elle prendrait à l'insu de celui qui l'entretient ${ }^{96}$. »

La transcendance métaphysique pensée par Lévinas se dégage de tous les mythes religieux:

La transcendance se distingue d'une union avec le transcendant, par participation. La relation métaphysique - l'idée de l'infini — relie au noumène qui n'est pas un numen. Ce noumène se distingue du concept de Dieu que possèdent les croyants des religions positives, mal dégagés des liens de la participation et qui s'acceptent comme plongés à leur insu, dans un mythe. L'idée de l'infini, la relation métaphysique est l'aube d'une humanité sans mythes ${ }^{97}$.

Elle est non idolâtrique:

Un Dieu qui vient à l'idée de cette manière [quand Dieu se révèle, il ne révèle rien de soi, mais il révèle l'homme à soi-même] est un Dieu dont la transcendance n'a rien d'idolâtre, et ce Dieu ne vient jamais à l'idée que dans les relations humaines quand elles se configurent comme relations de paix ${ }^{98}$.

\section{Redéfinissant ainsi la catégorie de religion}

Contre la religion chrétienne qui interprète le vécu du croyant comme une expérience d'être, de présence et d'immanence, et qui se représente l'expérience religieuse sans rompre avec la philosophie occidentale, Lévinas propose une troisième voie bien vivante entre un savoir de l'infini (philosophie) et une divinisation religieuse (religion):

Dans cette divinisation de l'Infini n'a-t-on pas perdu la divinité spécifiquement religieuse du Dieu qui permit à l'idée d'infini de dominer le rationalisme occidental? Pour une théologie qui fit de sa gnose l'objet même de cette gnose, toute relation avec l'Infini qui ne serait pas savoir passerait pour représentation sans concept, pour enfance de la pensée absolue. On peut se demander cependant si une autre voie n'est pas possible ${ }^{99}$.

Comment pourrait-on appeler cette troisième voie ? Étonnamment, au lieu d'abandonner le terme de "religion ", Lévinas le reprend: "L'ordre qui permet à la fois d'échapper au totalitarisme de la philosophie [...] mais aussi à l'anarchie des désirs individuels, cette vie qui est au-delà du livre, cette philosophie qui devient vie au lieu de devenir politique, c'est la religion ${ }^{100}$. » Mais ce terme - qui est à la fois ordre, philosophie et vie — est totalement redéfini :

96. Lévinas, Totalité et infini, p. 78.

97. Ibid., p. 75 .

98. Giuseppe Lissa, "Emmanuel Levinas: pour une transcendance non idolâtrique ", Pardès 2007/I, $\mathrm{n}^{\circ} 42,2007$, p. II 8 .

99. Lévinas, Altérité et transcendance, p. 89.

100. Lévinas, Difficile liberté, p. 280. 
- Ordre horizontal social, car la religion est une justice humaine dès lors qu'apparaît le tiers, représenté ici par l'État: «Justice comme raison d'être de l'État — voilà la religion ${ }^{101}$.»

- Ordre vertical séparé, car la religion est un rapport sans totalité avec la transcendance: "Nous réservons à la relation entre l'être ici-bas et l'être transcendant qui n'aboutit à aucune communauté de concept ni à aucune totalité — relation sans relation - le terme de religion ${ }^{102}$ » et "par opposition à la totalisation, nous l'avons appelée religion ${ }^{103}{ }^{\prime}$.

— Philosophie, car la religion est "comme surgissant, dans l'économie de l'être, au niveau même où y surgit la pensée philosophique ${ }^{104} »$.

— Vie enfin, car la religion est le souffle du vivant: "La religion, avant d'être une confession, est la pulsation même de la vie où Dieu entre en rapport avec l'homme, et l'homme avec le monde ${ }^{105}$.»

Par conséquent, Lévinas reprend le terme de religion, mais dans un tout autre sens que le sens courant: "Nous devons donc à Rosenzweig [...] le rappel d'une notion de religion toute différente de celle que le laicisme combat ${ }^{106}$.»

\section{Proche d'un athéisme}

Prise en ce sens, la religion se rapproche paradoxalement de l'athéisme, à condition de redéfinir également ce dernier terme. De nombreux connaisseurs de Lévinas disent d'ailleurs de la pensée lévinassienne qu'elle est a-religieuse, universelle, athée: "Il nous aura appris à reconnaître ce "Dieu qui vient à l'idée", même dans l'agnosticisme, même dans l'athéisme ${ }^{107}$." Lévinas ne cesse en effet de répéter combien sa pensée est nécessairement athée, et paradoxalement d'autant plus religieuse (au sens lévinassien) qu'elle restera athée:

Se rapporter à l'absolu en athée, c'est accueillir l'absolu apuré de la violence du sacré. [...] Seul un être athée peut se rapporter à l'Autre et déjà s'absoudre de cette relation. [...] la foi épurée des mythes, la foi monothéiste, suppose elle-même l'athéisme métaphysique. [...] L'athéisme conditionne une relation véritable avec vrai Dieu kath'auto ${ }^{108}$.

101. Ibid, p. 328 .

102. Ibid., p. 78-79.

103. Lévinas, Totalité et infini, p. I07.

104. Ibid., p. 28I.

105. Ibid., p. 284.

106. Ibid., p. 28 I. L'ouvrage de Franz Rosenzweig, L'étoile de la Rédemption paru en I92 I, a beaucoup influencé Lévinas.

107. De Saint Chéron, Entretiens avec Emmanuel Lévinas 1983-1994, p. I9.

108. Lévinas, Totalité et infini, p. 75. 
Pour Lévinas, l'athéisme n'est pas simplement la négation de l'existence de Dieu (sens courant). L'athéisme est redéfini pour signifier l'absolue séparation d'avec la transcendance et la non-participation au divin:

On peut appeler athéisme cette séparation si complète que l'être séparé se maintient tout seul dans l'existence sans participer à l'Être dont il est séparé - capable éventuellement d'y adhérer par la croyance. [...] L'âme - la dimension du psychique - accomplissement de la séparation, est naturellement athée. Par athéisme, nous comprenons ainsi une position antérieure à la négation comme à l'affirmation du divin, la rupture de la participation à partir de laquelle le moi se pose comme le même et comme moi ${ }^{109}$.

Lévinas se démarque d'une théologie thématisante qui (se) représente Dieu comme un objet. Dieu doit rester un «Dieu in-connu qui ne prend pas corps $^{110}$ ». C'est justement parce qu'il ne s'incarne pas que l'existence de Dieu suscite logiquement le scepticisme, l'agnosticisme, l'athéisme (au sens courant). Mais «il [le risque de l'athéisme] doit être couru. À travers lui seulement l'homme s'élève à la notion spirituelle du Transcendant ${ }^{111}$ ", car ce serait précisément dans son absence de manifestation que Dieu se montrerait le mieux.

\section{Et d'une spiritualité universelle}

Trouvant Dieu dans son absence, la pensée lévinassienne serait finalement moins religieuse (au sens courant) que spirituelle (étymologie renvoyant au souffle): "le spirituel ne se donne pas comme une substance sensible mais par l'absence ${ }^{112} »$. Lévinas fait bien remarquer qu' «on n'est pas entré dans les sables mouvants de l'expérience religieuse ${ }^{113}$ ", puisque cette dernière consiste au contraire en une recherche de présence.

Dès I96I, la pensée éthique de Lévinas s'affirme comme spiritualité: "L'éthique est l'optique spirituelle ${ }^{114}$.» Plus tard, dans une note de bas de page de De Dieu qui vient à l'idée, Lévinas s'interroge: "Dans la structure extraordinaire de l'idée de l'infini, l'à-Dieu ne signifie-t-il pas une intrigue spirituelle qui ne coïncide ni avec le mouvement marqué par la finalité, ni avec l'auto-identification de l'identité, telle qu'elle se déformalise dans la conscience de soi ? ${ }^{115}$ » Enfin en I992, Lévinas confirme: «L'éthique est aussi probablement la spiritualité même de l'âme [...]. Philosophie première ${ }^{116}$.»

109. Ibid., p. 52.

110. Lévinas, Altérité et transcendance, p. 54.

111. Lévinas, Difficile liberté, p. 34.

112. Ibid., p. 222.

113. Lévinas, De dieu qui vient à l'idée, p. I24.

114. Lévinas, Totalité et infini, p. 76.

115. Lévinas, De dieu qui vient à l'idée, p. I I.

116. Lévinas, Éthique comme philosophie première, p. I04. 
Cette spiritualité est universelle: «Il n'y a pas une seule chose dans une grande spiritualité qui soit absente d'une autre grande spiritualité ${ }^{117}$.

\section{Réalisée dans le judaïsme redéfini comme extrême conscience}

Or la spiritualité, ce rapport d'absence et de séparation absolue d'avec l'inconnu inconnaissable que décrit philosophiquement Lévinas, serait pleinement vécue dans le judaïsme, "monde spirituel infiniment plus complexe et plus raffiné que nos maladroites analyses ${ }^{118}$ ». Il nous faut donc revenir sur ce terme polysémique et mal compris:

Le mot «judaïsme» recouvre, de notre temps, des concepts très divers. Il désigne avant tout une religion - système de croyances, de rites et de prescriptions morales, fondées sur la Bible, sur le Talmud, sur la littérature rabbinique, souvent combinés avec la mystique ou la théosophie de la kabbale. [...] «Judaïsme » signifie dès lors une culture, résultat ou fondement de la religion, mais ayant un devenir propre. [...] Et cette sensibilité, et cette culture, et cette religion sont cependant perçues du dehors, comme les aspects d'une entité fortement caractérisée que l'on est embarrassé de classer. Nationalité ou religion? Civilisation fossilisée qui se survit, ou ferment d'un monde meilleur? Mystère d'Israël! ${ }^{119}$.

Pour Lévinas, le judaïsme n'est pas une religion (au sens courant) caractérisée par l'idolâtrie, l'extase, le sacré, le mythe et la religiosité, puisque «le judaïsme a désensorcelé le monde, a tranché sur cette prétendue évolution des religions à partir de l'enthousiasme et du sacré. Le judaïsme demeure étranger à tout retour offensif de ces formes d'élévation humaine. Il les dénonce comme l'essence de l'idolâtrie ${ }^{120}$.»

Il redéfinit le judaïsme auquel il est attaché comme "une religion d'adultes ${ }^{121}$ " "capable[s] de confiance en un Dieu absent ${ }^{122}$ ", "un Dieu d'adultes [qui] se manifeste précisément par le vide du ciel enfantin ${ }^{123}{ }$. Il s'agit donc d'un judaïsme libéré de toute position dogmatique et de toute religiosité qui, au lieu de chercher Dieu au Ciel, questionne les textes ${ }^{124}$ : «Le judaïsme, religion sans dogme, qui n'a pas vocation à convertir, est ouvert aux interprétations multiples de ses textes, renouvelées génération après génération ${ }^{125}$.» En ce sens, le judaïsme est pure rationalité et non foi: "Le

117. Lévinas, De dieu qui vient à l'idée, p. I48.

118. Emmanuel Lévinas, Quatre lectures talmudiques, Paris, Les Éditions de Minuit, I968, rééd. 2005, p. 25.

119. Lévinas, Difficile liberté, p. 48.

120. Ibid., p. 32.

121. Ibid., p. 27.

122. Ibid., p. 222.

123. Ibid., p. 220.

124. Le judaïsme rabbinique, religion à la fois aristocratique et démocratique, produit un «texte» polyphonique dépassant tout dialogue platonicien.

125. Klein, "Judaïsme, valeurs républicaines et laïcité », p. Io9. 
judaïsme de la raison doit prendre le pas sur le judaïsme de la prière; le juif du talmud doit prendre le pas sur le juif des Psaumes. [... ${ }^{126}$.»

Plus qu'une religion (sens courant), le judaïsme est une catégorie d'être. En 1957, lors d'un colloque, Lévinas dit que «le judaïsme n'est pas une religion, le mot n'existe pas en hébreu, il est beaucoup plus que cela, il est une compréhension de l'être ${ }^{127} »$. À propos du livre Étoile de la Rédemption de Rosenzweig qui l'a beaucoup influencé, Lévinas écrit:

Ce livre de philosophie générale est un livre juif qui fonde le judaisme d'une façon nouvelle: le judaïsme n'est plus seulement un enseignement dont les thèses seraient vraies ou fausses, l'existence juive [...] elle-même est un événement essentiel de l'être, l'existence juive est une catégorie de l'être ${ }^{128}$.

Le judaïsme qui déborde l'ancienne catégorie de religion est précisément cette spiritualité que Lévinas cherche à décrire et à fonder:

Le judaïsme est à l'étroit dans le concept de religion tel que le formule la sociologie, il ne se limite pas aux démarches que la psychologie religieuse suppose dans l'âme du croyant. J'en appellerai à des témoignages [...] ils attestent une spiritualité étrangère à la catégorie reçue de religion ${ }^{129}$.

Le judaïsme réaliserait précisément l'idéal philosophique, permettant de vivre la transcendance, que la philosophie occidentale vise rationnellement depuis toujours sans jamais l'atteindre. Le judaïsme réussirait ainsi là où la philosophie échoue. Cela fait d'ailleurs dire à $\mathrm{F}$. Ciaramelli que la perspective de Lévinas est réaliste plutôt qu'idéaliste ${ }^{130}$. Cependant, le judaïsme est une spiritualité "accessible à l'analyse ${ }^{131}$ ».

Lévinas définit le judaïsme de façon plurielle:

— Le judaïsme est un rapport laïc à une transcendance, puisque celle-ci est absolument séparée de l'être humain qui la pense: «Humanisme hébraïque. Sa notion demeure laïque ${ }^{132}$ ».

— Le judaïsme est d'abord et surtout «une extrême conscience ${ }^{133}$ » située «au carrefour de la foi et de la logique ${ }^{134}$ ", logée précisément dans ce lieu improbable qu'est l'écart entre la croyance et la raison.

— Le judaïsme est "une attitude concrète, une expérience absolue de l'infiniment autre ${ }^{135}$ » grâce à l'affranchissement du savoir, à la révélation antérieure à

126. Lévinas, Difficile liberté, p. 404.

127. Malka, Lévinas, la vie et la trace, p. I42.

128. Lévinas, Difficile liberté, p. 275.

129. Ibid., p. 370.

130. Ciaramelli, "Le rôle du judaïsme dans l'œuvre de Levinas ».

131. Lévinas, Difficile liberté, p. 48.

132. Ibid., p. 407

133. Ibid., p. 19.

134. Ibid., p. 407.

135. Banon, «Levinas, penseur juif ou juif qui pense», p. 3 . 
la pensée du discours et de l'hétérogénéité, à la pratique des commandements et des rites.

— Le judaïsme est aussi un enseignement: «Le judaïsme nous enseigne une transcendance réelle, une relation avec Celui que l'âme ne peut contenir et sans Lequel elle ne peut, en quelque façon, se tenir elle-même ${ }^{136}$.»

— Le judaïsme passe par la Thora: «La physionomie particulière du judaïsme: le rapport entre Dieu et l'homme [...] une relation entre esprits, par l'intermédiaire d'un enseignement, par la Thora ${ }^{137}$ ».

— Le judaïsme passe par l'interprétation talmudique: «ce qui pour nous juifs demeure l'essentiel: la constitution du Talmud. [...] S'il n'y avait pas de Talmud, il n'y aurait pas de juifs aujourd'hui ${ }^{138}$ ». Et: "Le judaïsme, ce n'est pas la Bible, c'est la Bible vue à travers le Talmud, à travers la sagesse, l'interrogation et la vie religieuse rabbiniques ${ }^{139}$."

Le judaïsme ainsi compris conduit à des questions essentielles pour la philosophie.

\section{5. ...Et conduisant à des questions philosophiques essentielles}

La pensée de Lévinas qui s’inspire de ce judaïsme (ainsi redéfini) redécouvre d'abord sa proximité avec la philosophie occidentale: "À l'égard du divin qu'ils [les dieux numineux et nombreux] incarnent, il [le monothéisme juif] n'est qu'athéisme. Ici, le judaïsme se sent extrêmement proche de l'Occident, je veux dire de la philosophie ${ }^{140}$. " Mais il est plus large et dépasse cette philosophie: "Le judaïsme n'est pas simplement une affaire de juifs et d'antisémites, c'est proprement une affaire de pensée qui convoque le monde, l'identité, l'histoire et excède toute philosophie stricte ${ }^{141}$. " Le judaïsme n'est donc pas équivalent à la philosophie, mais il en est une source grâce aux textes talmudiques: "Si le Talmud n'est pas la philosophie, ses traités sont une source éminente de ces expériences dont se nourrissent les philosophies ${ }^{142}$.

Philosophie et judaïsme se rejoignent dans leur quête de vérité, mais tandis que la première la cherche dans le savoir et l'ontologie, le second la cherche dans la relation éthique: "Lévinas lui-même indique l'importance de la référence au judaïsme eu égard à la question de la vérité », car "le judaïsme permet de poser la question de la vérité en dépassant la référence ontologique et en privilégiant la structure éthique ${ }^{143}$ ».

136. Lévinas, Difficile liberté, p. 35.

137. Ibid., p. 22 I.

138. Ibid., p. 265.

139. Poirié, Emmanuel Lévinas. Essai et entretiens, p. I 56.

140. Lévinas, Difficile liberté, p. 33.

141. Gérard Bensussan, «Sartre, Lévinas et les Juifs. Phénoménologie de l'antisémitisme et ontologie du judaïsme ", Les Temps modernes, $\mathrm{n}^{\circ} 664$, 201 I, p. I 50.

142. Lévinas, Quatre lectures talmudiques, p. I2.

143. Ciaramelli, "Le rôle du judaïsme dans l'œuvre de Levinas", p. 582. 
Finalement, qu'apporte cette pensée inspirée du judaïsme à la philosophie? D'après nous, elle pose deux questions philosophiques essentielles portant sur le sens:

I. La question éthique du sens de l'être humain en particulier: "Question par excellence ou la question de la philosophie. Non pas: pourquoi l'être plutôt que rien, mais comment l'être se justifie ${ }^{144}$.» Quel est le sens de l'existence humaine? Comment conjuguer éthiquement la liberté du sujet et le droit à exister d'autrui ? Comment articuler la responsabilité transcendante utopique dans la relation duelle et la justice humaine imparfaite lorsqu'intervient le tiers?

2. La question du sens en général: "Que signifie l'intelligibilité de l'intelligible, la signification du sens, que signifie la raison? C'est là sans doute la question préalable de l'humain épris de sens, la question préalable de la philosophie. Ou la question même de la philosophie ${ }^{145}$." Qu'est-ce qui est accessible à la raison (donc à la philosophie occidentale) et qu'est-ce qui ne l'est pas? Quel est le sens de cet écart entre raison et croyance, entre philosophie occidentale et judaïsme?

Ce faisant, Lévinas introduit «la diversion judaïque au cœur même de la philosophie ${ }^{146} »$. Réciproquement, la posture philosophique de Lévinas renouvelle le judaïsme en y (ré)introduisant ${ }^{147}$ la raison philosophique:

Monsieur Lévinas nous incitait de la sorte, dans l'esprit de Maïmonide, à nous mettre à la philosophie pour mieux nous arranger de notre perplexité juive, peut-être mieux l'assumer comme distinction humaine. Présumer en toutes circonstances de la pertinence - philosophique - de l'Écriture et de ses commentaires. Fidélité première et dernière, peut-être devoir religieux de tout philosophe juif. La philosophie ne représentait pas un fourvoiement, elle était au contraire requise pour lever les incertitudes et enrichir le judaïsme ${ }^{148}$.

En fin de compte, le judaïsme pourrait bien représenter l'altérité de la raison philosophique ${ }^{149}$. Lévinas ne les envisage d'ailleurs pas l'un sans l'autre, «ne se mettant à parler grec que parce qu'il ne s'entendait qu'à un discours sur le judaïsme qui s'articulerait selon les rets de la raison philosophique ${ }^{150}$ ».

Comment Lévinas parvient-il à ces problématiques essentielles?

144. Lévinas, Éthique comme philosophie première, p. I09.

145. Lévinas, De dieu qui vient à l'idée, p. I73.

146. David Banon, "Lévinas talmudiste", in Emmanuel Lévinas, Philosophe et pédagogue, Paris, Éd. du Nadir de l'Alliance Israélite Universelle, I998, p. 33.

147. La pensée de Lévinas s'inspire d'un ancien hégélien (Franz Rosenzweig) et d'un grand kantien (Hermann Cohen).

148. Bouganim, "Lévinas pédagogue », p. 59.

149. Alain David, "Lévinas et la phénoménologie. L'enjeu de la sainteté ", Les Temps modernes, $\mathrm{n}^{\circ} 664$, 20II, p. 94-I I 8.

150. Bouganim, "Lévinas pédagogue ", p. 73. 


\section{Grâce à une méthode: I'herméneutique talmudique}

Parallèlement à la phénoménologie, Lévinas pratique l'herméneutique talmudique. Elle est l'analyse et l'interprétation philosophiques des textes fondamentaux du judaïsme (Bible, Thora, Talmud). Proposant des leçons "d'un style particulier ${ }^{151}$ ", Lévinas s'efforce dans un premier temps «en fidèle" «d'entrer d'abord dans le langage de la tradition non philosophique attachée à la compréhension religieuse des écritures juives ${ }^{152}$ "; dans un deuxième temps, il cherche une justification phénoménologique au verset étudié: "Une vérité philosophique ne peut pas se baser sur l'autorité du verset. Il faut que le verset soit phénoménologiquement justifié. Mais le verset peut permettre la recherche d'une raison ${ }^{153}$.»D. Banon parle de «la méthode et son renversement " : l'exégèse pour faire parler les textes talmudiques tout en se préservant de l'idolâtrie puis «l'envolée métaphysique souvent sous-tendue par l'analyse phénoménologique des options philosophiques défendues par chaque rabbi ${ }^{154}$ ».

Cette envolée suggère le plaisir généré par l'interprétation des textes, au moment de la construction et du partage d'une nouvelle intelligibilité, c'est-à-dire à l'instant de la création d'un sens nouveau, processus dont Lévinas donne plusieurs métaphores: "frotter le texte pour en faire jaillir le sang ${ }^{155}$ », "faire sortir un peu d'eau de ce texte désertique ${ }^{156}$ », "comme le marteau qui frappe la roche et en fait jaillir des étincelles» ou encore «comme la braise sur laquelle on souffle et qui devient flamme ${ }^{157}$ ». Conformément à sa problématique du sens en général explicitée précédemment, nous comprenons que Lévinas cherche à construire du sens pour créer le chaînon manquant entre judaïsme et philosophie.

Comment s'y prend-il?

Le rabbin Daniel Epstein, traducteur en hébreu des «lectures talmudiques » parle, à propos de Lévinas, d'une «lecture phénoménologique» du Talmud, d'une sorte de «Midrash phénoménologique " qui consisterait à lire chaque passage en l'inscrivant dans le contexte plus général de la "Souguia ", à faire apparaître les horizons cachés du texte, ce qui a été oublié, ce qui a été ignoré, ce qui a été offusqué. C'était effectivement sa manière. Qui devait sans doute autant à sa formation phénoménologique [...] qu'à la fréquentation du redoutable Chouchani [maître talmudique] ${ }^{158}$.

151. Malka, Lévinas, la vie et la trace, p. I39.

152. Poirié, Emmanuel Lévinas. Essai et entretiens, p. I3I.

153. Ibid., p. I3 I.

154. Banon, "Lévinas talmudiste", p. 40.

155. Lévinas, Quatre lectures talmudiques, p. IO2.

156. Ibid., p. I 8 .

157. Malka, Lévinas, la vie et la trace, p. I4I.

158. Ibid., p. I37. 
Le verset n'est pour Lévinas qu'un exemple particulier dont on peut dégager une question philosophique universelle: "Il y a des situations où, dans le verset - qui joue toujours initialement le rôle d'illustration ou de suggestion -, surgit une idée qui prend une certaine force du fait que ce contexte religieux amène un accent philosophique ${ }^{159}$." À partir du cas illustré dans le verset, l'exégèse lévinassienne tend vers le questionnement et la vérité philosophiques: "une lecture du Talmud qui ne se limiterait ni à la philologie, ni à la piété à l'égard d'un passé “cher mais périmé”, ni à l'acte religieux d'adoration; mais une lecture en quête de problèmes et de vérités $[\ldots]^{160}$ » universels: "Nous prenons le texte talmudique et le judaïsme qui s'y manifeste pour enseignants et non pas pour un tissu mythogène de survivances. [...] Les vues universelles qu'il s'agit de dégager du particularisme apparent $[\ldots]$ voilà le but dominant de notre exégèse ${ }^{161}$. »

Ni méthode historique ni analyse structuraliste, la démarche lévinassienne est une méthode rationaliste qui résiste à la tentation d'élaborer un savoir sur le divin :

Le rationalisme de la méthode ne consiste pas, Dieu merci, à remplacer Dieu par Être Suprême ou par Nature ou — comme le font certains jeunes hommes en Israël - par Peuple juif ou Classe ouvrière. Il consiste d'abord à se méfier de tout ce qui, dans les textes étudiés, pourrait passer pour une information sur la vie de Dieu, pour une théosophie ${ }^{162}$.

Le rationalisme est la caractéristique première de la méthode lévinassienne: "Mon effort consiste toujours à dégager de ce langage théologique des significations qui s'adressent à la raison ${ }^{163}$.» Ce caractère rationnel de la démarche garantit "la liberté et le non-dogmatisme dans l'exégèse ${ }^{164}$ ». Lévinas revendique cette liberté apprise de son Maître: «Il [M. Chouchani] a rendu impossible à jamais l'accès dogmatique purement fidéiste, ou même théologique, au Talmud. Notre essai doit attester cette recherche de liberté, sinon une liberté conquise ${ }^{165}$.» Cette liberté d'interprétation frôle parfois la subversion: "Lecture subversive, qui n'avait rien de pieux, qui s'en défendait même, et se revendiquait d'une liberté conquise ${ }^{166}$." Cette lecture «révolutionnaire» ne suivait ni n'imposait de dogmes: «Il laissait aux auditeurs le soin de déduire. C'était bien sûr un discours construit, orienté, intelligent, mais jamais il n'imposait son interprétation ${ }^{167}$.» La lecture

159. Poirié, Emmanuel Lévinas. Essai et entretiens, p. I32.

160. Lévinas, Quatre lectures talmudiques, p. 23.

161. Ibid., p. I4-I 5 .

162. Ibid., p. 33 .

163. Ibid., p. 33 .

164. Ibid., p. I4-I 5 .

165. Ibid., p. 22.

166. Malka, Lévinas, la vie et la trace, p. I38.

167. Ibid., p. I40. 
lévinassienne restait ouverte au dialogue et au débat pour co-construire un sens rationnel universel:

Nous pouvons donc aborder hardiment ce texte religieux, mais qui se prête, d'une façon si merveilleusement naturelle, au langage philosophique. Il n'est pas dogmatique, il vit de discussions et de colloques. Le théologique reçoit ici une signification morale d'une remarquable universalité où se reconnaît la raison $^{168}$.

La méthode lévinassienne peut être qualifiée d'herméneutique paradigmatique multidimensionnelle et dialectique:

Ainsi ces signes - versets bibliques, objets, personnes, situations, rites fonctionneront comme signes parfaits. [...] Signes parfaits, irremplaçables et, dans ce sens - purement herméneutique - signes sacrés, lettres sacrées, saintes écritures. [...] Jamais l'esprit ne donne congé à la lettre qui le révèle. [...] Les possibilités de signifier à partir d'un objet concret libéré de son histoire - ressource d'une méthode de pensée que nous avons appelée paradigmatique - sont innombrables. Requérant l'usage de facultés spéculatives peu communes, elles se déroulent dans un espace multidimensionnel. La dialectique du Talmud prend un rythme océanique [...] un mouvement incessant de va-et-vient ${ }^{169}$.

Alors cette méthode si particulière doit-elle encore être qualifiée de « religieuse » ? Dans ses entretiens, Lévinas répond à cette question sous forme de boutade: «Si on estime que cette deuxième manière d'engendrer la pensée [en partant de la Bible] est religieuse, je suis penseur religieux!170" Tout dépend effectivement de la définition que l'on donne au terme "religieux».

\section{Conclusion}

Le soupçon et le rejet viennent souvent de la méconnaissance et de l'incompréhension. Notre contribution visait donc à faire œuvre pédagogique en donnant une vue d'ensemble argumentée et détaillée du dessein parfaitement clair, cohérent et logique d'Emmanuel Lévinas. Le philosophe voulait en effet clarifier, différencier et ré-articuler les catégories en jeu: philosophie, Dieu, religion, athéisme, judaïsme. Ce faisant, nos résultats s'inscrivent dans la continuité de certains travaux de recherche. Notre contribution a en effet tiré le fil de l'hypothèse rapidement évoquée par J. A. Barash selon laquelle Lévinas nourrissait "très exactement cette double ambition: critiquer les catégories traditionnelles et en redéfinir de nouvelles ${ }^{171} »$. Elle a également tenté de faire le pas de côté préconisé par F. Mies: "Ceci [le fait que la pensée lévinassienne ne s'oppose pas à la laïcité] ne peut s'expliquer, évi-

168. Lévinas, Quatre lectures talmudiques, p. 34.

169. Ibid., p. 20.

170. Poirié, Emmanuel Lévinas. Essai et entretiens, p. I34.

171. Barash, «Emmanuel Lévinas: penseur du religieux ?», p. I I9. 
demment, que si le terme "religieux" change de sens. Or la façon lévinassienne d'entendre "Dieu" suppose un pas de côté ${ }^{172}$. " Notre analyse permet de proposer la synthèse suivante:

\begin{tabular}{|c|c|c|}
\hline Catégorie & Sens courant (d'après Lévinas) & Sens «lévinassien» \\
\hline Philosophie & $\begin{array}{l}\text { Philosophie classique } \\
\text { occidentale: } \\
\text { - amour la sagesse } \\
\text { - avec priorité au Vrai } \\
\text { - insatisfaite d'elle-même } \\
\text { (manque, désir de savoir, } \\
\text { besoin de réponse), } \\
\text { - ontologique (Penser = être), } \\
\text { - immanente, } \\
\text { - passant par la connaissance. }\end{array}$ & $\begin{array}{l}\text { Philosophie de l'amour: } \\
\text { - sagesse de l'amour } \\
\text { - avec priorité au Bien } \\
\text { - satisfaite d'elle-même (union, } \\
\text { fusion, communion), } \\
\text { - au-delà de (antérieurement à) } \\
\text { l'ontologie, } \\
\text { - métaphysique et transcendante, } \\
\text { - passant par la relation éthique } \\
\text { (Visage d'autrui). }\end{array}$ \\
\hline Dieu & $\begin{array}{l}\text { Transcendance révélée: } \\
\text { Dieu pensé en termes } \\
\text { ontologiques comme un objet, } \\
\text { thématisé, représenté, } \\
\text { personnifié, prenant corps ou } \\
\text { dans le Ciel. }\end{array}$ & $\begin{array}{l}\text { Transcendance non révélée: } \\
\text { Dieu = idée de l'infini, séparation } \\
\text { absolue d'avec l'être humain qui } \\
\text { la pense, extériorité, absence de } \\
\text { corrélation dans la pensée entre } \\
\text { le sujet et l'objet, absence de } \\
\text { contenu, courbure intersubjective, } \\
\text { hétéronomie, kath'auto, } \\
\text { interpellation éthique, assignation } \\
\text { à responsabilité, le tiers et } \\
\text { l'Illéité. }\end{array}$ \\
\hline Religion & $\begin{array}{l}\text { - Relation à Dieu } \\
\text { - en termes de participation et } \\
\text { de totalité, } \\
\text { - rapport vertical, } \\
\text { - foi, violence du sacré, mythe, } \\
\text { idolâtrie, extase, religiosité, } \\
\text { dogmes religieux } \\
\text { - expérience religieuse } \\
\text { - sanctification }\end{array}$ & $\begin{array}{l}\text { - Rapport à Dieu } \\
\text { - sans participation ni totalité } \\
\text { (oubli de soi dans la relation), } \\
\text { - rapport horizontal, } \\
\text { - religion d'adultes, rationnelle, } \\
\text { non dogmatique, sans sacré, ni } \\
\text { mythe, ni idolâtrie, spiritualité } \\
\text { athée universelle précédant le } \\
\text { savoir }\end{array}$ \\
\hline
\end{tabular}

172. Françoise Mies, "Religions, laïcité et écritures saintes selon Emmanuel Lévinas. Réflexions pour notre temps ", Nouvelle revue théologique, 20I I/2, vol. I33, 20 I I, p. 270. 


\begin{tabular}{|c|c|c|}
\hline $\begin{array}{l}\text { Religion } \\
\text { (suite) }\end{array}$ & & $\begin{array}{l}\text { - conscience extrême, } \\
\text { - «sainteté } \text { : hyperbole, excès } \\
\text { pour dire amour, responsabilité, } \\
\text { condition d'otage, passivité } \\
\text { devant la justice, acceptation } \\
\text { de la mort, valeur, idéal } \\
\text { humaniste, utopie, possibilité } \\
\text { du sacrifice. }\end{array}$ \\
\hline Athéisme & $\begin{array}{l}\text { Athéisme (religieux): } \\
\text { - croyance en la non-existence } \\
\text { de Dieu } \\
\text { - ou négation de l'existence } \\
\text { de Dieu }\end{array}$ & $\begin{array}{l}\text { Athéisme (métaphysique): } \\
\text { - antérieur à toute croyance (en } \\
\text { l'existence ou en la non- } \\
\text { existence de Dieu). } \\
\text { - Séparation absolue entre la } \\
\text { transcendance (idée de l'infini) } \\
\text { et l'être qui tente de la penser. }\end{array}$ \\
\hline Judaïsme & $\begin{array}{l}\text { Religion - institution - } \\
\text { système de croyances, de rites, } \\
\text { de prescriptions morales - } \\
\text { mystique - kabbale - } \\
\text { culture } \\
\text { — nationalité - «civilisation } \\
\text { fossilisée» }\end{array}$ & $\begin{array}{l}\text { Religion en tant que rapport à } \\
\text { l'infini, spiritualité, catégorie } \\
\text { d'être, religion d'adultes devant } \\
\text { un Ciel vide, quête de vérité } \\
\text { passant par l'interprétation des } \\
\text { textes talmudiques. }\end{array}$ \\
\hline
\end{tabular}

Remarquons alors que la différenciation des catégories apparaît très clairement, tant dans leur sens «lévinassien» que dans leur sens courant. Remarquons aussi et surtout que leur articulation change du tout au tout:

- dans leur sens courant, la philosophie occidentale insatisfaite et toujours avide de savoir s'oppose radicalement à une religiosité faite de dogmes, de sacré, de mythes, d'idolâtrie envers un Dieu personnifié. Le discours philosophique a en effet toujours voulu connaître et absorber le Dieu dont parle la Bible, pour détruire la transcendance révélée.

— au contraire, dans leur sens «lévinassien", la religion (au sens de rapport séparé de l'être humain à une transcendance impensable, universelle, spirituelle, vivante, inspirée du judaïsme et surgissant de la relation éthique) fonde une sagesse de l'amour, la seule vraie philosophie, et inversement la seule qui puisse interpréter et garder vivante une religion d'adultes.

Un changement paradigmatique apparait alors, passant d'une opposition totale et violente entre philosophie et religion (au sens courant) à une douce et enrichissante interdépendance entre philosophie et religion (au sens «lévinassien»).

En proposant une phénoménologie de l'idée de l'Infini, le philosophe ancre la transcendance dans les relations humaines, redéfinit toutes les caté- 
gories en jeu, propose un changement paradigmatique et pose de façon visionnaire des questions essentielles dont les solutions proposées nous semblent intéressantes :

I. la problématique du sens en général qui se loge dans l'écart entre ce qui est intelligible (investi par la philosophie occidentale) et ce qui ne l'est pas (investi jusqu'à présent par les croyances), qui nécessite des moyens (l'herméneutique) et des lieux (les textes) pour remettre en œuvre un processus de création de sens;

2. la problématique du sens de l'être humain en particulier qui se loge dans l'écart entre sa liberté et le droit à exister d'autrui, qui nécessite elle aussi des moyens (l'éthique et la justice) et des lieux (la relation et la société) pour mettre en œuvre une sagesse de l'amour.

Nos sociétés contemporaines aux prises avec la radicalisation violente et nos écoles en recherche d'une éthique éducative n'ont-elles pas besoin d'urgence de cette re-création de sens et de cette sagesse de l'amour? 